\title{
Association of Preoperative De Ritis Ratio (Aspartate Amino Transferase/Alanine Amino Transferase) and Tumour Histology in Patients with Primary Bladder Cancer
}

\author{
(D) Abdullah Gül1', (D) Nazım Abdulkadir Kankılıç² \\ 1 University of Health Sciences Turkey, Bursa Yüksek Ihtisas Training and Research Hospital, Clinic of Urology, Bursa, Turkey \\ 2 University of Health Sciences Turkey, Van Training and Research Hospital, Clinic of Urology, Van, Turkey
}

\begin{abstract} considered significant. between De Ritis ratio and MIBC ( $p=0.036)$, and $G$ of tumour ( $p=0.009)$. associated with muscle invasion (T2) and high tumour G of BC.

Keywords: Amino transferases, bladder cancer, de ritis ratio, grade, stage

\section{Introduction}

Bladder cancer (BC), a common genitourinary tract cancer, is the sixth most frequently diagnosed cancer in the United States, and also the third most common type of cancer in male population of Turkey $(1,2,3)$. Globally, it has an average standardized mortality rate of 3.2 per 100,000 men and 0.9 per 100,000 women (1). In approximately $75 \%-85 \%$ of newly diagnosed BC cases, tumour is infiltrated in the mucosa [stage Ta and carcinoma in situ (CIS)] or submucosa (stage T1) and are classified as nonmuscle invasive BC (NMIBC) for therapeutic purposes $(4,5)$. For treatment of $B C$, transurethral resection of the bladder tumour (TUR-BT) is performed and, thereafter, intravesical instillations
\end{abstract}

Objective: De Ritis ratio, defined as the ratio of serum aspartate amino transferase (AST) and alanine amino transferase (ALT), has been demonstrated to be an independent prognostic marker in bladder cancer (BC) patients who underwent radical cystectomy. We aimed to investigate the relationship between preoperative De Ritis ratio and tumour histology prior to transurethral resection of bladder tumour (TUR-BT) in patients newly diagnosed with BC.

Materials and Methods: Data of the patients who underwent TUR-BT between May 2016 and August 2019 at two tertiary hospitals were evaluated, retrospectively. The patients were grouped as either non-muscle invasive $B C$ (NMIBC) or muscle invasive $B C(M I B C)$, and as grade (G) 1,2 and 3 according to pathological and histological G, respectively. Further, patients were classified as low, intermediate or high risk according to risk group stratification. Demographic features of the patients including age and gender were recorded. Pre-treatment biochemical indices including serum levels of AST and ALT were recorded. P value $<0.05$ was

Results: Mean age of patients (156) included in this study was $67.10 \pm 10.15$. Exactly 104 (66.7\%) patients were placed in the NMIBC group, while the remaining $52(33.3 \%)$ patients were placed in the MIBC group. De Ritis ratio was lower in the NMIBC group than in the MIBC group ( $p=0.014)$. Also, there was a significantly difference in De Ritis ratio between $G 1$ and $G 3$ carcinomas $(p=0.040)$. According to receiver operating characteristics analysis, the cut-off threshold of De Ritis ratio for clinically predicting MIBC and high-G carcinoma was 1.21 and 1.20, respectively. Based on Spearman's correlation analysis, a weak positive correlation was found

Conclusion: This study demonstrated that an increase in De Ritis ratio, which can be easily determined from the peripheral blood in preoperative period, may be

are incorporated in order to prevent disease recurrence and progression to muscle invasive $B C$ (MIBC). However, radical cystectomy $(\mathrm{RC})$ is a preferred treatment strategy for localized MIBC $(6,7)$. Moreover, some patients with high risk NMIBC may undergo RC prior to muscle invasion (8).

Amino transferase enzymes such as aspartate amino transferase (AST) and alanine amino transferase (ALT) are leaked into circulation from liver cells as indication for hepatocellular damage. De Ritis (9) put forward an hypothesis in 1957 that the ratio of these enzymes could be used to predict viral hepatitis. Successfully, the term De Ritis ratio (calculated by dividing AST by $A L T$ ) took its place in the literature. Till date, this ratio has been employed in a lot of research as biomarker for diagnosis,

Cite this article as: Gül A, Kankilıç NA. Association of Preoperative De Ritis Ratio (Aspartate Amino Transferase/Alanine Amino Transferase) and Tumour Histology in Patients with Primary Bladder Cancer. Bull Urooncol 2020;19(3):136-140

Address for Correspondence: Abdullah Gül, University of Health Sciences Turkey, Bursa Yuksek İhtisas Training and Research Hospital, Clinic of Urology, Bursa, Turkey Phone: +90224295 5000 E-mail: dr abdullahgul@hotmail.com ORCID-ID: orcid.org/0000-0003-4002-4659 
treatment and follow-up of, not only hepatic disorders, but also non-hepatic diseases $(10,11,12,13,14)$. Today, it is popularly employed for prognosis of many cancers $(15,16,17,18,19,20)$. In literature, elevated preoperative De Ritis ratio was regarded as an independent indicator of poor prognosis in patients who underwent RC $(18,21,22)$.

Therefore, we aim to investigate whether De Ritis ratio can be employed to predict tumour histology and risk group prior to TUR-BT in patients newly diagnosed with BC.

\section{Materials and Methods}

After obtaining ethics committee approval of institutional review board (approval number: 2019/21, date: 05.08.2019), we conducted a cross-sectional study including patients who underwent TUR-BT for primary BC between May 2016 and August 2019 at two tertiary hospitals. Age, gender, grade and stage of tumour, risk group of tumour, and preoperatively evaluated serum AST-ALT levels were obtained from electronic database and surgery notes in the hospitals. Patients whose records were not sufficient for the evaluation, as well as those with liver metastasis, were excluded from this study. In addition, patients undergoing any form of treatment which may influence the level of amino transferase enzymes; patients with hepatic disorders such as chronic hepatitis, liver cirrhosis or other concurrent cancers, and patients with inadequate muscle tissue in the resected histopathology specimens were excluded from this study. Classification of tumour stage and tumour grade was according to the 2009 tumor node metastasis classification updated in 2017 (23) and 1973 World Health Organization (WHO) grading system (24), respectively. Where a second resection was required according to the criteria specified in European Association of Urology (EAU) Guidelines (25), we performed a second TUR-BT. We accepted findings regarding upstaging or upgrading of pathological results of second TURBT. Since some pathologists examined specimens of TUR-BT only according to WHO 1973 classification for reporting BC histology, we could not evaluate the results according to 2004 WHO grading system. According to the 2019 EAU Guidelines, risk group stratification was low, intermediate or high risk (25). Further, patients were grouped into two (NMIBC and MIBC) according to the pathological stage, and into three according histological grade. We further grouped patients with NMIBC into three based on the risk group stratification.

\section{Statistical Analyses}

Statistical analysis was done using IBM SPSS version 21 (IBM Corp., Armonk, NY, USA). Variables were expressed as means and standard deviations. Student's independent t-test was used to compare differences between two groups, while One-way analysis of variance followed by Tukey Post-hoc test was utilized to compare differences between three groups. Chi-square test and Fisher's Exact test were used for comparison of qualitative data. The capacity of De Ritis ratio in predicting muscle invasion and high grade of tumour was determined using receiver operating characteristics (ROC) curve analysis. Spearman's correlation analysis was used to evaluate relationship between De Ritis ratio and the groups. Also, we used a linear regression model to identify independent predictors of tumour histology. $\mathrm{P}<0.05$ was considered significant.

\section{Results}

After the exclusion of patients (67) who did not meet inclusion criteria, a total of 156 patients with primary BC were included in the study. Of the total patients included, 104 (66.7\%) patients had NMIBC, while the remaining 52 (33.3\%) patients had MIBC (stage T2 tumour). Descriptive statistics and mean values for all patients are summarised in Table 1. Mean age and De Ritis ratio were significantly different between patients with NMIBC and MIBC (Table 2). Based on T stage, mean age of patients with stage Ta tumour was significantly lower than that of patients with stage $\mathrm{T} 2$ tumour $(63.58 \pm 10.02$ vs $70.50 \pm 10.03, \mathrm{p}=0.004)$. Similarly, patients with stage Ta tumour had lower De Ritis ratio than patients with stage $\mathrm{T} 2$ tumour $(1.17 \pm 0.31$ vs $1.47 \pm 0.69$, $\mathrm{p}=0.027)$. Based on the tumour grade, there were significant differences between grade 1 and grade 3 carcinomas in terms of age and De Ritis ratio ( $p=0.011$ and $p=0.040$, respectively). All the parameters between the risk group of patients with NMIBC were non-significantly different ( $p>0.05$ ) (Table 3$)$. According to ROC curve, the cut-off value of preoperative De Ritis ratio for predicting MIBC was 1.21 , with $56 \%$ sensitivity and $55 \%$ specificity [area under curve (AUC) $=0.603,95 \%$ confidence interval $(\mathrm{Cl})=0.503-0.702, \mathrm{p}=0.037$ ] (Figure 1). On the other

\begin{tabular}{|c|c|c|c|c|c|}
\hline Tumor histology & n (\%) & Age (year) & AST (U/L) & ALT (U/L) & AST/ALT \\
\hline \multicolumn{6}{|l|}{ Stage } \\
\hline $\mathrm{Ta}$ & $46(29.9)$ & $63.58 \pm 10.02$ & $19.28 \pm 4.76$ & $17.78 \pm 7.87$ & $1.17 \pm 0.31$ \\
\hline $\mathrm{T} 1$ & $54(34.6)$ & $67.25 \pm 9.45$ & $21.61 \pm 5.01$ & $19.16 \pm 7.43$ & $1.25 \pm 0.46$ \\
\hline $\mathrm{T} 2$ & $52(33.3)$ & $70.50 \pm 10.03$ & $19.78 \pm 5.53$ & $16.02 \pm 8.66$ & $1.47 \pm 0.69$ \\
\hline Tis & $4(2.2)$ & $61.50 \pm 9.98$ & $18.50 \pm 1.73$ & $18 \pm 4.76$ & $1.05 \pm 0.22$ \\
\hline \multicolumn{6}{|l|}{ Grade } \\
\hline G1 & 49 (31.4) & $63.93 \pm 11.74$ & $19.77 \pm 5.10$ & $19.18 \pm 8.71$ & $1.15 \pm 0.43$ \\
\hline G2 & $10(6.4)$ & $64.10 \pm 3.84$ & $23.10 \pm 3.54$ & $18.90 \pm 2.13$ & $1.21 \pm 0.07$ \\
\hline G3 & $97(62.2)$ & $69.02 \pm 9.29$ & $20.17 \pm 5.24$ & $16.79 \pm 7.90$ & $1.38 \pm 0.57$ \\
\hline Total & 156 & $67.10 \pm 10.15$ & $20.23 \pm 5.13$ & $17.68 \pm 7.98$ & $1.29 \pm 0.52$ \\
\hline
\end{tabular}




\begin{tabular}{|c|c|c|c|}
\hline Variables & NMIBC $(n=104)$ & MIBC $(n=52)$ & $p$ \\
\hline Age (year) & $65.41 \pm 9.83$ & $70.50 \pm 10.03$ & 0.003 \\
\hline \multicolumn{4}{|l|}{ Gender } \\
\hline Male $(n=136,87.2 \%)$ & $90(86.5 \%)$ & $46(88.5 \%)$ & \multirow{2}{*}{0.735} \\
\hline Female $(n=20,12.8 \%)$ & $14(13.5 \%)$ & $6(11.5 \%)$ & \\
\hline AST (U/L) & $20.46 \pm 4.94$ & $19.79 \pm 5.53$ & 0.460 \\
\hline $\operatorname{ALT}(\mathrm{U} / \mathrm{L})$ & $18.51 \pm 7.53$ & $16.02 \pm 8.66$ & 0.082 \\
\hline AST/ALT & $1.21 \pm 0.39$ & $1.47 \pm 0.69$ & 0.014 \\
\hline
\end{tabular}

Table 3. Comparison of data in patients with non-muscle invasive bladder cancer in terms of risk group stratification

\begin{tabular}{|c|c|c|c|c|}
\hline Data & $\begin{array}{l}\text { Low risk } \\
(n=19)\end{array}$ & $\begin{array}{l}\text { Intermediate risk } \\
(n=19)\end{array}$ & $\begin{array}{l}\text { High risk } \\
(n=66)\end{array}$ & $p$ \\
\hline Age (year) & $61.89 \pm 11.15$ & $63.10 \pm 9.57$ & $67.09 \pm 9.23$ & NS \\
\hline \multicolumn{5}{|l|}{ Gender } \\
\hline Male $(n=90)$ & $16(84.2 \%)$ & 15 (78.9\%) & 59 (89.4\%) & \multirow{2}{*}{ NS } \\
\hline Female $(n=14)$ & $3(15.8 \%)$ & $4(21.1 \%)$ & $7(10.6 \%)$ & \\
\hline AST (U/L) & $19.68 \pm 5.96$ & $20.05 \pm 3.59$ & $20.80 \pm 4.98$ & NS \\
\hline $\operatorname{ALT}(\mathrm{U} / \mathrm{L})$ & $20 \pm 11.11$ & $17.36 \pm 3.74$ & $18.40 \pm 7.12$ & NS \\
\hline AST/ALT & $1.14 \pm 0.41$ & $1.17 \pm 0.19$ & $1.24 \pm 0.43$ & NS \\
\hline
\end{tabular}



Figure 1. ROC curve for prediction of $\mathrm{MIBC}(\mathrm{AUC}=0.603(95 \% \mathrm{Cl}=0.503-0.702$, $\mathrm{p}=0.037$ )

ROC: Receiver operating characteristics, MIBC: Muscle invasive bladder cancer, AUC: Area under curve, $\mathrm{Cl}$ : Confidence interval hand, for prediction of high-grade BC, ROC cut-off value was 1.20 , with $58 \%$ sensitivity and $66 \%$ specificity (AUC $=0.617,95 \%$ $\mathrm{Cl}=0.528-0.705, \mathrm{p}=0.015$ ) (Figure 2). According to Spearman correlation analysis, De Ritis ratio had a weak positive correlation with muscle invasion of $B C(r=+0.236, p=0.036)$ and grade of tumour $(r=+0.208, p=0.009)$. On the other hand, there was no correlation between De Ritis ratio and risk groups $(p=0.471)$. According to regression analysis, patients' age did not influence the De Ritis ratio.

\section{Discussion}

Surgeons are poised to forecast histopathologic features of tumours prior to operation. The ability to clinically predict the invasiveness or grade of new tumours is often aid the decision on tumour management. According to European Organization for Research and Treatment of Cancer risk scores, BC patients are primarily grouped into NMIBC or MIBC, and into risk groups in order to predict 1 and 5 year recurrence and progression in terms recurrence rate, number of tumours, tumour size, $\mathrm{T}$ stage, grade, and simultaneous CIS presence. In this regard, patients with NMIBC are classified into low, intermediate and high risk groups for the purpose of treatment (26). Many studies have evaluated blood biomarkers and cystoscopy of tumour in view of predicting the histopathological stage and grade of $\mathrm{BC}$ $(27,28,29)$. Vartolomei et al. (27) revealed in their meta-analysis that NMIBC patients with higher neutrophil-to-lymphocyte ratio, assessed prior to TUR-BT, have greater risk of disease recurrence and progression.

Tumour cells require excessive uptake of glucose (for glycolysis) in order to produce sufficient adenosine triphosphate, which



Figure 2. ROC curve for prediction of high-grade BC (AUC $=0.617(95 \%$ $\mathrm{Cl}=0.528-0.705, \mathrm{p}=0.015$ )

ROC: Receiver operating characteristics, AUC: Area under curve, BC: Bladder cancer, $\mathrm{Cl}$ : Confidence interval 
is referred to as the Warburg (30) effect. Increased glycolysis results in certain changes in mitochondrial activities and, in turn, alterations in activity of nicotinamide adenine dinucleotiderelated enzyme and glucose transporters (31). BC is regarded as one of the glucose-reliant malignant tumours $(32,33)$. This was also revealed by a study in which fluorescence microscopy images of BC cells show utilization of glucose (34). AST and ALT are needed as catalyst in glutamine metabolism. AST is also main component of malate-aspartate shuttle pathway $(35,36)$. They are highly implicated in cellular metabolism and tumour proliferation. AST is a less liver-specific enzyme and increases much more in cancer compared to ALT $(12,15,37)$. Therefore, De Ritis ratio might, not only is a predictor of glucose consumption in cancers, but also a predictor of tumour aggressiveness.

De Ritis ratio has been frequently used as a prognostic marker in many cancers (38). In uro-oncology, it was investigated in several types of cancer including BC, prostate cancer, renal cell carcinoma and urinary tract urothelial carcinoma $(15,16,18,19,21,22)$. All the studies on BC showed an elevated preoperative De Ritis ratio, which was related to poor prognosis in patients who underwent RC $(18,21,22)$. However, these studies focused on predicting the survival of previously diagnosed $B C$ patients. To the best of our knowledge, our study is the first to predict tumour histology in patients newly diagnosed with BC. We found that higher preoperative De Ritis ratio was associated with higher stage and grade of $\mathrm{BC}$. We observed a statistically significant correlation between high and low grade of BC, and between NMIBC and MIBC. In addition, our study revealed that De Ritis ratio was an age independent predictor of tumour histology.

A study conducted in Turkey revealed that patients with elevated De Ritis ratio (with a cut-off value of 1.3) had worse diseasespecific and overall survival outcomes (18). Similar results were reported by Ha et al. (21). In our study, the cut-off value of preoperative De Ritis ratio for predicting muscle invasive and high-grade BC was 1.21 (sensitivity $=56 \%$, specificity $=55 \%$ ) and 1.20 (sensitivity $=58 \%$, specificity $=66 \%$ ), respectively. We demonstrated in our study that preoperatively evaluated AST/ ALT value $>1.21$ was shown to be useful in predicting T2 or upstage in BC patients. Despite the low levels (Figures 1,2), the AUC, sensitivity and specificity of De Ritis ratio in predicting both muscle invasion and high-grade BC were similar to aforementioned studies. In addition, we showed that patients with stage $\mathrm{Ta}$ and $\mathrm{G1}$ tumour were younger than those with higher stage and grade of tumour. Age and tumour stage, along with an elevated De Ritis rate, were found to be independent prognostic factors for cancer and overall survival of patients with BC who underwent RC $(18,22)$.

\section{Study Limitations}

In spite of the significant and new contributions of this study to literature, it has some inherit drawbacks. Firstly, this study was limited by its retrospective nature and relatively small sample size. Secondly, the type of $B C$ was not classified. This was due to low number of patients with adenocarcinoma and squamous cell carcinoma in urinary bladder, and less than $10 \%$ of all BC patients, paralleling with literature (39). Regardless of the cancer type, we analysed the data obtained based on tumour stage and grade. Also, MIBC patients were not evaluated based on metastasis since we aimed to investigate the relationship between preoperative De Ritis ratio and primary $\mathrm{BC}$ and tumour histology prior to TUR-BT. If there was any distant organ metastasis of the tumour in MIBC group, it might affect the serum level of the amino transferase enzymes. Nevertheless, a prospective study including all pathological tumour stage with a long follow-up period will help to clarify this gap. Lastly, we could not evaluate the results according to WHO 2004/2016 grading system. However, the WHO 2004/2016 grading system is not superior to the WHO 1973 used in the present study (40).

\section{Conclusion}

AST and ALT are rapidly, cheaply and reproducibly measured serum biomarkers before performance of surgery. Considering the necessity for intensive and sustainable treatment for $B C$, we suggest that preoperatively assessed De Ritis ratio will be a helpful and useful predicting tool after diagnosis, treatment, and during follow-up of BC. However, this study needs to be validated by well-designed large-scale prospective clinical studies.

\section{Acknowledgements}

Publication: The results of the study were not published in full or in part in form of abstracts.

Contribution: There is not any contributors who may not be listed as authors.

Conflict of Interest: No conflict of interest was declared by the authors.

Financial Disclosure: The authors declared that this study received no financial support.

\section{Ethics}

Ethics Committee Approval: After obtaining ethics committee approval of institutional review board (approval number: 2019/21, date: 05.08.2019), we conducted a cross-sectional study including patients who underwent TUR-BT for primary BC between May 2016 and August 2019 at two tertiary hospitals.

Informed Consent: Retrospective study.

Peer-review: Externally and internally peer-reviewed.

\section{Authorship Contributions}

Concept: A.G., N.A.K., Design: N.A.K., Data Collection or Processing: N.A.K., Analysis or Interpretation: A.G., Literature Search: A.G., N.A.K., Writing: A.G.

\section{References}

1. Ferlay J, Soerjomataram I, Dikshit R, et al. Cancer incidence and mortality worldwide: sources, methods and major patterns in GLOBOCAN 2012. Int J Cancer 2015; 136:359-386.

2. Siegel RL, Miller KD, Fedewa SA, et al. Colorectal cancer statistics, 2017. CA Cancer J Clin 2017;67:177-193.

3. Aydin S, Boz MY. Rapid changes in the incidence of urinary system cancers in Turkey. Turk J Urol 2015;41:215-220.

4. Anastasiadis $A$, de Reijke TM. Best practice in the treatment of nonmuscle invasive bladder cancer. Ther Adv Urol 2012;4:13-32.

5. Comperat E, Larre S, Roupret $M$, et al. Clinicopathological characteristics of urothelial bladder cancer in patients less than 40 years old. Virchows Arch 2015;466:589-594. 
6. Hautmann RE, Abol-Enein $\mathrm{H}$, Hafez $\mathrm{K}$, et al. World Health Organization (WHO) consensus conference in bladder cancer. Urology 2007;69(Suppl 1):17-49.

7. Stein JP, Lieskovsky G, Cote R, et al. Radical cystectomy in the treatment of invasive bladder cancer: long-term results in 1,054 patients. J Clin Oncol 2001;19:666-675.

8. Herr HW, Sogani PC. Does early cystectomy improve the survival of patients with high risk superficial bladder tumors? J Urol 2001;166:1296-1299.

9. De Ritis F, Coltorti M, Giusti G. An enzymic test for the diagnosis of viral hepatitis: the transaminase serum activities. Clinica Chimica Acta 1957;2:70-74.

10. Steininger M, Winter M-P, Reiberger T, et al. De-Ritis Ratio Improves Long-Term Risk Prediction after Acute Myocardial Infarction. J Clin Med 2018;7:474.

11. Gao F, Chen C, Lu J, et al. De Ritis ratio (AST/ALT) as an independent predictor of poor outcome in patients with acute ischemic stroke. Neuropsychiatr Dis Treat 2017;13:1551-1557.

12. Zoppini G, Cacciatori V, Negri C, et al. The aspartate aminotransferaseto-alanine aminotransferase ratio predicts all-cause and cardiovascular mortality in patients with type 2 diabetes. Medicine 2016;95:e4821.

13. Rief $P$, Pichler $M$, Raggam $R$, et al. The AST/ALT (De-Ritis) ratio: $A$ novel marker for critical limb ischemia in peripheral arterial occlusive disease patients. Medicine 2016;95:e3843.

14. Botros M, Sikaris K. The De Ritis Ratio: the test of time. Clin Biochem Rev 2013;34:117-130.

15. Canat L, Ataly HA, Agalarov S, et al. The effect of AST/ALT (De Ritis) ratio on survival and its relation to tumor histopathological variables in patients with localized renal cell carcinoma. Int Braz J Urol 2018;44:288-295.

16. Wang H, Fang K, Zhang J, et al. The significance of De Ritis (aspartate transaminase/alanine transaminase) ratio in predicting pathological outcomes and prognosis in localized prostate cancer patients. Int Urol Nephrol 2017;49:1391-1398.

17. Liu C, Jia B-s, Zou B-w, et al. Neutrophil-to-lymphocyte and aspartateto-alanine aminotransferase ratios predict hepatocellular carcinoma prognosis after transarterial embolization. Medicine 2017;96:e8512.

18. Gorgel SN, Kose O, Koc EM, et al. The prognostic significance of preoperatively assessed AST/ALT (De Ritis) ratio on survival in patients underwent radical cystectomy. Int Urol Nephrol 2017;49:1577-1583.

19. Cho YH, Hwang JE, Chung HS, et al. The De Ritis (aspartate transaminase/alanine transaminase) ratio as a predictor of oncological outcomes in patients after surgery for upper urinary tract urothelial carcinoma. Int Urol Nephrol 2017;49:1383-1390.

20. Takenaka Y, Takemoto N, Yasui T, et al. Transaminase activity predicts survival in patients with head and neck cancer. PloS one 2016;11:e0164057.

21. Ha YS, Kim SW, Chun SY, et al. Association between De Ritis ratio (aspartate aminotransferase/alanine aminotransferase) and oncological outcomes in bladder cancer patients after radical cystectomy. BMC Urol 2019;19:10.

22. Gökçen K, Kıraç E, Gökçen P, et al. Preoperative AST/ALT (De Ritis) Ratio as a Prognostic Factor in a Cohort of Patients who underwent radical cystectomy. Cumhuriyet Medical Journal 2018;40:299-307.
23. TNM classification of malignant tumors. 8th ed. Brierley JD, editor. Wiley-Blackwell; 2017.

24. Mostofi FK, Sobin LH, Torloni H, Organization WH. Histological typing of urinary bladder tumours. 1973.

25. Compérat PG, Mostafid AH, Palou J, et al. EAU Guidelines on nonmuscle-invasive bladder cancer. Babjuk M, editor. EAU 2019.

26. Sylvester RJ, van der Meijden AP, Oosterlinck W, et al. Predicting recurrence and progression in individual patients with stage Ta T1 bladder cancer using EORTC risk tables: a combined analysis of 2596 patients from seven EORTC trials. Eur Urol 2006;49:466-465.

27. Vartolomei MD, Porav-Hodade D, Ferro $M$, et al. Prognostic role of pretreatment neutrophil-to-lymphocyte ratio (NLR) in patients with non-muscle-invasive bladder cancer (NMIBC): A systematic review and meta-analysis. Urol Oncol 2018;36:389-399.

28. During VA, Sole GM, Jha AK, et al. Prediction of histological stage based on cystoscopic appearances of newly diagnosed bladder tumours. Ann R Coll Surg Engl 2016;98:547-551.

29. Bhindi B, Hermanns T, Wei Y, et al. Identification of the best complete blood count-based predictors for bladder cancer outcomes in patients undergoing radical cystectomy. Br J Cancer 2016;114:207-212.

30. Warburg O. On the origin of cancer cells. Science 1956;123:309-314.

31. Dorward A, Sweet S, Moorehead R, Singh G. Mitochondrial contributions to cancer cell physiology: redox balance, cell cycle, and drug resistance. J Bioenerg Biomembr 1997;29:385-392.

32. Yun SJ, Jo SW, Ha YS, et al. PFKFB4 as a prognostic marker in nonmuscle-invasive bladder cancer. Urol Oncol 2012;30:893-899.

33. Chang SG, Lee JH, Hong DH, et al. Comparison of glucoseconsumption and thymidine-incorporation endpoints in histocultured human superficial bladder tumors. Anticancer Res 1994;14:77-83.

34. Whyard T, Waltzer WC, Waltzer D, Romanov V. Metabolic alterations in bladder cancer: applications for cancer imaging. Exp Cell Res 2016;341:77-83.

35. Vander Heiden MG, Cantley LC, Thompson CB. Understanding the Warburg effect: the metabolic requirements of cell proliferation. Science 2009;324:1029-1033.

36. Cairns RA, Harris IS, Mak TW. Regulation of cancer cell metabolism. Nat Rev Cancer 2011;11:85-95.

37. Botros M, Sikaris KA. The de ritis ratio: the test of time. Clin Biochem Rev 2013;34:117-130.

38. Wu J, Chen L, Wang Y, et al. Prognostic value of aspartate transaminase to alanine transaminase (De Ritis) ratio in solid tumors: a pooled analysis of 9,400 patients. Onco Targets Ther 2019;12:5201-5213.

39. Murta-Nascimento C, Schmitz-Drager BJ, Zeegers MP, et al. Epidemiology of urinary bladder cancer: from tumor development to patient's death. World J Urol 2007;25:285-295.

40. Soukup V, Capoun O, Cohen D, et al. Prognostic Performance and Reproducibility of the 1973 and 2004/2016 World Health Organization Grading Classification Systems in Non-muscle-invasive Bladder Cancer: A European Association of Urology Non-muscle Invasive Bladder Cancer Guidelines Panel Systematic Review. Eur Urol 2017;72:801-813. 